PROCEEDINGS OF THE

AMERICAN MATHEMATICAL SOCIETY

Volume 131, Number 10, Pages 3275-3283

S 0002-9939(03)06856-4

Article electronically published on February 6, 2003

\title{
POINCARÉ DUALITY IN P.A. SMITH THEORY
}

\author{
CHRISTOPHER ALLDAY, BERNHARD HANKE, AND VOLKER PUPPE \\ (Communicated by Paul Goerss)
}

\begin{abstract}
Let $G=S^{1}, G=\mathbb{Z} / p$ or more generally $G$ be a finite $p$-group, where $p$ is an odd prime. If $G$ acts on a space whose cohomology ring fulfills Poincaré duality (with appropriate coefficients $k$ ), we prove a mod 4 congruence between the total Betti number of $X^{G}$ and a number which depends only on the $k[G]$-module structure of $H^{*}(X ; k)$. This improves the well known mod 2 congruences that hold for actions on general spaces.
\end{abstract}

\section{INTRODUCTION AND STATEMENT OF RESULTS}

Let $X$ be a finite dimensional connected CW complex and let $k$ be a commutative ring with unit. We say that $X$ is a Poincaré duality space over $k$ (we will often write $k$-PD space instead) of formal dimension $n$, if $H^{*}(X ; k)$ is a finitely generated $k$-module and if there is a class $\nu \in H_{n}(X ; k)$ such that

$$
H^{*}(X ; k) \rightarrow H_{n-*}(X ; k), \quad c \rightarrow c \cap \nu
$$

is an isomorphism. Note that if $k$ is a field, this is equivalent to requiring that

$$
H^{*}(X ; k) \times H^{*}(X ; k) \stackrel{\cup}{\longrightarrow} H^{*}(X ; k) \stackrel{\nu}{\longrightarrow} k
$$

is a nonsingular pairing (viewing $\nu$ as an element in $\left.\operatorname{Hom}\left(H^{*}(X ; k), k\right)\right)$.

Let $G$ denote the group $S^{1}$ (with its usual topology) or $\mathbb{Z} / p$, where $p$ is an odd prime number. Let $k=\mathbb{Q}$, if $G=S^{1}$, and $k=\mathbb{F}_{p}$, if $k=\mathbb{Z} / p$. By a well known result proven independently by Chang-Skjelbred in [2] and Bredon in [3], each component of the fixed point set of a finite dimensional $G$-CW complex $X$ fulfills Poincaré duality over $k$, if this is property holds for $X$. By now there are several further versions and variants of proofs of this result $([1,5,8])$. In this paper, we will use certain consequences of Poincaré duality to deduce relations between the total Betti number of $X^{G}$ and the $k[G]$-module $H^{*}(X ; k)$.

Theorem 1. Let $G=S^{1}$ and let $X$ be a finite dimensional connected $G-C W$ complex such that $X$ is a $\mathbb{Q}-P D$ space of formal dimension $n$. If

- $n$ is even or

- $n=2 m+1, X^{G} \neq \emptyset, H^{i}(X ; \mathbb{Q})=0$ for $0<i \leq m$, $i$ even,

Received by the editors September 20, 2001 and, in revised form, May 3, 2002.

2000 Mathematics Subject Classification. Primary 57S10, 57P10, 55N10; Secondary 55N91.

Key words and phrases. Group action, Betti number, Poincaré duality space.

The second author holds a DFG research grant. He thanks the University of Notre Dame for its hospitality during the work on this paper. 
then the following congruence holds:

$$
\operatorname{dim}_{\mathbb{Q}} H^{*}\left(X^{G} ; \mathbb{Q}\right) \equiv \operatorname{dim}_{\mathbb{Q}} H^{*}(X ; \mathbb{Q}) \quad \bmod 4 .
$$

If $G=S^{1} \times \ldots \times S^{1}$ and if $X$ is a finite dimensional connected $G$-CW complex with finitely many orbit types that fulfills Poincaré duality over $\mathbb{Q}$, then an analogue of Theorem 1 holds. This is true, because in this case we can choose a subcircle $S^{1} \subset G$, such that $X^{S^{1}}=X^{G}$.

A version of Theorem 1 for actions of $G=\mathbb{Z} / p$, where $p$ is an odd prime, can be proven under additional assumptions on the space $X$. It turns out that one also has to take into account the fact that now the induced $G$-action on $H^{*}\left(X ; \mathbb{F}_{p}\right)$ might be nontrivial. Using a spectral sequence argument together with results from [6], we show:

Theorem 2. Let $G=\mathbb{Z} / p$ (where $p$ is an odd prime) and let $X$ be a finite dimensional connected $G-C W$ complex such that $X$ is an $\mathbb{F}_{p}-P D$ space of formal dimension $n$. Furthermore, assume that $H^{*}\left(X ; \mathbb{Z}_{(p)}\right)$ does not contain $\mathbb{Z} / p$ as a direct summand. Then, we get a decomposition as graded $\mathbb{F}_{p}[G]$-modules

$$
H^{*}\left(X ; \mathbb{F}_{p}\right)=F^{*} \oplus T^{*} \oplus R^{*},
$$

where $F^{*}$ is a free $\mathbb{F}_{p}[G]$-module, $T^{*}$ is a trivial $\mathbb{F}_{p}[G]$-module and $R^{*}$ is a direct sum of $\mathbb{F}_{p}[G]$-modules of the form $\operatorname{ker} \epsilon$, where $\epsilon: \mathbb{F}_{p}[G] \rightarrow \mathbb{F}_{p}$ is the augmentation map. If

- $n$ is even or

- $n=2 m+1, X^{G} \neq \emptyset, T^{i}=0$ for $0<i \leq m$, $i$ even, $R^{i}=0$ for $0<i \leq m$, $i$ odd,

then the following congruence holds:

$$
\operatorname{dim}_{\mathbb{F}_{p}} H^{*}\left(X^{G} ; \mathbb{F}_{p}\right) \equiv \operatorname{dim}_{\mathbb{F}_{p}} T^{*}+\frac{1}{p-1} \operatorname{dim}_{\mathbb{F}_{p}} R^{*} \bmod 4 .
$$

Theorem 1 was first proved by A. Sikora in his Ph.D. thesis. This and Theorem 2 for the case that $R^{*}=0$ and $H^{*}(X ; \mathbb{Z})$ does not contain any $p$-torsion are also contained in his paper [9], where a somewhat different line of argument is used.

It is well known that the cited result by Bredon, Chang and Skjelbred immediately generalizes to actions of finite $p$-groups $G$. This is achieved by applying induction on the order of $G$ and using the fact that every finite (nontrivial) $p$ group contains a normal subgroup of order $p$. This procedure can be applied in our situation as well and shows the following result.

Theorem 3. Let $G$ be a finite p-group (where $p$ is an odd prime) and let $X$ be a finite dimensional connected $G$-CW complex that is an $\mathbb{F}_{p}-P D$ space of even formal dimension. If $p>\operatorname{dim} H^{*}\left(X ; \mathbb{F}_{p}\right)$, then

$$
\operatorname{dim}_{\mathbb{F}_{p}} H^{*}\left(X^{G} ; \mathbb{F}_{p}\right) \equiv \operatorname{dim}_{\mathbb{F}_{p}} H^{*}\left(X ; \mathbb{F}_{p}\right) \quad \bmod 4
$$

If we impose more restrictions on $X$ and on the group operation, then using a result from [7, we can use a similar induction argument for comparing rational Betti numbers:

Theorem 4. Let $G$ be a finite p-group (where $p$ is an odd prime) and let $X$ be a finite connected simplicial complex with $G$ acting simplicially such that $X$ is an 
even dimensional orientable $\mathbb{Z}_{(p)}$-homology manifold. Furthermore, assume that $p>\operatorname{dim} H^{*}\left(X ; \mathbb{F}_{p}\right)$. Then

$$
\operatorname{dim}_{\mathbb{Q}} H^{*}\left(X^{G} ; \mathbb{Q}\right) \equiv \operatorname{dim}_{\mathbb{Q}} H^{*}(X ; \mathbb{Q}) \quad \bmod 4 .
$$

In the case of smooth actions, this result can be proven without making use of 7.

In Section 5, we will show by examples that none of the additional assumptions in the second part of Theorem 1 and Theorem 2 can be dropped.

We would like to thank the referee for some helpful comments and suggestions.

\section{Algebraic Preliminaries}

For our later applications to spectral sequences, it will be useful to provide a notion of Poincaré duality for bigraded algebras.

Definition 1. Let $k$ be a field and let $A^{*, *}$ be a $(\mathbb{Z} / 2 \times \mathbb{N})$-bigraded associative and graded commutative $k$-algebra with unit that is finitely generated as $k$-vector space. (The graded commutativity is required with respect to the total $\mathbb{Z} / 2$-grading, where $A^{i, j}$ has total degree $i+j \bmod 2$.) Then

i. We call $A^{*, *}$ connected, if $A^{0,0} \cong k$.

ii. $A^{*, *}$ is a $k$-Poincaré duality algebra of formal dimension $n$, if there is a surjective linear map $\phi: A^{*, *} \rightarrow k$ (called orientation) with $\phi\left(A^{i, j}\right)=0$ for $(i, j) \neq(0, n)$ such that the bilinear form

$$
\zeta: A^{*, *} \times A^{*, *} \stackrel{\text { mult. }}{\longrightarrow} A^{*, *} \stackrel{\phi}{\longrightarrow} k
$$

is nondegenerate.

Note that in a connected Poincaré duality algebra of formal dimension $n$, we have $A^{0, n} \cong k$. The following elementary fact provides a connection between the Euler characteristic and the total Betti number of Poincaré duality algebras of even formal dimension.

Lemma 1. Let $A^{*, *}$ be a Poincaré duality algebra of even formal dimension over a field $k$. If chark $\neq 2$, then

$$
\operatorname{dim}_{k} A^{*, *} \equiv \chi\left(A^{*, *}\right) \quad \bmod 4
$$

where the Euler characteristic is calculated using the total $\mathbb{Z} / 2$-grading of $A$.

Proof. Let $2 m$ be the formal dimension of $A^{*, *}$. Using the induced total $\mathbb{Z} / 2$ grading of $A^{*, *}$, we claim that the dimension of $A^{\text {odd }}$ is even, which obviously proves the lemma. For all $i$, we get isomorphisms

$$
\begin{aligned}
A^{0,2 i+1} & \cong \operatorname{Hom}\left(A^{0,2 m-2 i-1}, k\right), \\
A^{1,2 i} & \cong \operatorname{Hom}\left(A^{1,2 m-2 i}, k\right)
\end{aligned}
$$

by Poincaré duality. Hence, $A^{0,2 i+1}$ and $A^{0,2 m-2 i-1}$, respectively $A^{1,2 i}$ and $A^{1,2 m-2 i}$, have the same dimension. Furthermore, if $m$ is even, the module $A^{1, m}$ carries a skew nonsingular form by Poincaré duality and therefore has even dimension, as char $k \neq 2$. The same is true for $A^{0, m}$, if $m$ is odd. 
Proposition 1. Let $\left(A^{*, *}, \delta\right)$ be a connected $(\mathbb{Z} / 2 \times \mathbb{N})$-graded differential algebra over the field $k$ with a differential $\delta$ of arbitrary bidegree, but lowering the second grading parameter and acting as a derivation. Furthermore, assume that $A^{*, *}$ is a Poincaré duality algebra of formal dimension $n$. Then, after taking homology, we get either $H\left(A^{*, *}, \delta\right)=0$ or $H\left(A^{*, *}, \delta\right)$ is again a connected Poincaré duality algebra of formal dimension $n$.

Proof. Assume that $H\left(A^{*, *}\right) \neq 0$. This implies that the unit of $A$ (that generates $\left.A^{0,0}\right)$ is not in the image of $\delta$. Let $c \in A^{0, n} \cong k$ be a generator. Assume that $\delta(c)=$ $x \neq 0$. By Poincaré duality, we then find an element $y \in A^{*, *}$ with $c=y x \in A^{0, n}$. We therefore get

$$
0 \neq x=\delta(c)=\delta(y x)=\delta(y) x
$$

which implies that $\delta(y)$ is a generator of $A^{0,0}$, contrary to what we said before. Hence, because $c$ is not hit by $\delta$, any orientation $\phi$ of $A$ induces a surjective linear map $H^{*, *}(A) \rightarrow k$. To complete the proof, observe that by the derivation property of $\delta$, we have

$$
\zeta(\operatorname{ker} \delta, \operatorname{im} \delta)=0,
$$

using the bilinear form $\zeta$ from Definition 1 As

$$
\operatorname{dim}_{k} \operatorname{ker} \delta+\operatorname{dim}_{k} \operatorname{im} \delta=\operatorname{dim}_{k} A^{*, *},
$$

we may conclude that $\operatorname{ker} \delta$ is exactly the orthogonal complement of im $\delta$ with respect to $\zeta$. This proves that the induced bilinear form on $H^{*, *}(A)$ is nonsingular.

Proposition 2. Let $\left(A^{*, *}, \delta\right)$ be as in Proposition 1. Additionally, we assume that char $k \neq 2$, the formal dimension of $A^{*, *}$ is an odd number $2 m+1$, the differential $\delta$ has odd (total) degree, lowering the second grading parameter in $A^{*, *}$ and $A^{0, i}=0$ for $0<i \leq m$, $i$ even, $A^{1, i}=0$ for $0<i \leq m$, $i$ odd. If $H\left(A^{*, *}\right) \neq 0$, then

$$
\operatorname{dim}_{k} A^{*, *} \equiv \operatorname{dim}_{k} H\left(A^{*, *}, \delta\right) \bmod 4 .
$$

Proof. Let $Z^{*}=\operatorname{ker} \delta \subset A^{*}$ denote the cycles in $A^{*}$ with respect to $\delta$ (here we use the total $\mathbb{Z} / 2$ grading, again). By our assumption on $A^{*}$, the even dimensional part $H^{e v}\left(A^{*}, \delta\right)$ of the homology of $A^{*}$ with respect to $\delta$ coincides with $Z^{e v}$; cf. the proof of Proposition 1 Now we define a bilinear form

$$
\gamma: A^{e v} \times A^{e v} \rightarrow k, \quad(x, y) \rightarrow \phi(x \cdot \delta(y)),
$$

using an orientation $\phi$ of $A$. It is easy to check that $\gamma$ induces a well defined, nonsingular and skew symmetric form on $A^{e v} / Z^{e v}$, hence this vector space has even dimension over $k$ (using the fact that char $k \neq 2$ ). As the Euler characteristic of $A^{*}$ and of $H\left(A^{*}\right)$ are equal, we get the equation

$$
A^{e v}-H^{e v}\left(A^{*}\right)=A^{\text {odd }}-H^{o d d}\left(A^{*}\right) .
$$

Therefore, the number $\operatorname{dim} A^{*}-\operatorname{dim} H^{*}\left(A^{*}\right)$ is divisible by 4 .

\section{Proof of Theorems 1, 3 and 4}

Here, when applying the results from the last section, we usually forget about the first grading parameter of $A^{*, *}$ and use the $\mathbb{N}$-grading by the second parameter. Part of the argument is based on the following well known fact (cf. [1], Exercise (3.29)). 
Lemma 2. Let $G=S^{1}$ or $G=\mathbb{Z} / p$ and let $X$ be a finite dimensional $G-C W$ complex such that $H^{*}(X ; \mathbb{Z})$ is a finitely generated $\mathbb{Z}$-module. Then

i. $\chi\left(X^{G}\right)=\chi(X)$, if $G=S^{1}$.

ii. $\chi\left(X^{G}\right)=\Lambda(g)$, if $G=\mathbb{Z} / p$ and $g \in G, g \neq 1$.

Here, $\Lambda(g)$ denotes the Lefschetz number

$$
\Lambda(g)=\sum_{i}(-1)^{i} \operatorname{trace}\left(g_{*}: H_{i}(X ; \mathbb{Q}) \rightarrow H_{i}(X ; \mathbb{Q})\right),
$$

regarding $g$ as a map $X \rightarrow X$.

If we set $A^{0,1}=0$ and $A^{*, 0}=H^{*}(X ; \mathbb{Q})$, respectively $A^{*, 0}=H^{*}\left(X^{G} ; \mathbb{Q}\right)$, then Lemma 1 and Lemma 2 give the following sequence of equations:

$$
\operatorname{dim} H^{*}\left(X^{G} ; \mathbb{Q}\right) \equiv \chi\left(X^{G}\right)=\chi(X) \equiv \operatorname{dim} H^{*}(X ; \mathbb{Q}) \bmod 4 .
$$

This shows the first part of Theorem 1

The proof of Theorem 3 proceeds by induction on the order of $G$. Assume $|G| \neq 1$ and choose a normal subgroup $H \subset G, H \cong \mathbb{Z} / p$ that exists by group theory. Each component of $X^{H}$ is an $\mathbb{F}_{p}$-PD space of even formal dimension by the theorem of Bredon-Chang-Skjelbred. As $\operatorname{dim} H^{*}(X ; \mathbb{Q})<p$ by our assumption on $p$, the induced action of $H$ on $H^{*}(X ; \mathbb{Q})$ is trivial: Let $V$ be a rational vector space of dimension smaller than $p-1$ and let $f$ be a linear endomorphism of $V$ with $f^{p}=$ id. Then the minimal polynomial of $f$ in $\mathbb{Q}[x]$ must divide $x^{p}-1$. The last polynomial splits over $\mathbb{Q}[x]$ into $(x-1)$ and an irreducible factor of degree $p-1$. Because the minimal polynomial of $f$ has degree at most $\operatorname{dim} V$, it must therefore be equal to $x-1$. This covers all cases, where $H^{*}(X ; \mathbb{Q})$ is not concentrated in degree 0 . In the remaining case, the assertion follows from the fact that $H^{0}(X ; \mathbb{Q})$ is a permutation module.

Altogether, for $1 \neq h \in H$, we obtain

$$
\Lambda(h)=\chi(X) .
$$

By Lemma 1 and Lemma 2 above, we get

$$
\operatorname{dim} H^{*}\left(X^{H} ; \mathbb{F}_{p}\right) \equiv \chi\left(X^{H}\right)=\chi(X) \equiv \operatorname{dim} H^{*}\left(X ; \mathbb{F}_{p}\right) \bmod 4 .
$$

(Note that the Euler characteristic does not depend on the coefficient field used.) For the induction step, observe that by Smith theory

$$
\operatorname{dim} H^{*}\left(F ; \mathbb{F}_{p}\right) \leq \operatorname{dim} H^{*}\left(X ; \mathbb{F}_{p}\right)<p
$$

for each component $F$ of $X^{G}$. Furthermore, the group $G^{\prime}=G / H$ has order less than the order of $G$ and each component of $X^{H}$ is invariant under the induced $G^{\prime}$-action by our assumption on $p$. Using the theorem of Bredon-Chang-Skjelbred, each component of $X^{H}$ is again an $\mathbb{F}_{p}$-PD space of even formal dimension. Hence the induction hypothesis applies to each component of $X^{H}$. The proof of Theorem 3is now complete.

For the proof of Theorem 4 , we recall the following fact.

Proposition 3 ([7], Proposition 13). Let $G=\mathbb{Z} / p$ act simplicially on a finite simplicial complex $X$ that is an orientable $\mathbb{Z}_{(p)}$-homology manifold. If $F \subset X$ is a component of the fixed point set $X^{G}$, then $F$ is an orientable $\mathbb{Z}_{(p)}$-homology manifold of even codimension in $X$. 
Using this fact, Theorem 4 follows from Theorem 3 by observing that the total Betti numbers with either $\mathbb{F}_{p}$ or $\mathbb{Q}$ coefficients of a space are congruent modulo 4 , if this space fulfills Poincaré duality both over $\mathbb{Q}$ and over $\mathbb{F}_{p}$ and has even formal dimension for both fields of coefficients. This follows by the independence of the Euler characteristic of the coefficient field and by using Lemma 1 twice.

For the proof of the second part of Theorem 1 we consider the cohomological Leray-Serre spectral sequence $\left(E_{r}, \delta_{r}\right)$ for the Borel fibration

$$
X \rightarrow X_{G}=E G \times_{G} X \rightarrow B G
$$

with coefficients in $\mathbb{Q}$. For the $E_{2}$-term of this spectral sequence as a module over $H^{*}(B G ; \mathbb{Q}) \cong \mathbb{Q}[t]$, where $t \in H^{2}(B G ; \mathbb{Q})$ is a generator, we get

$$
E_{2}^{*, \mu} \cong H^{\mu}(X ; \mathbb{Q}) \otimes \mathbb{Q}[t] .
$$

Because all the differentials in the spectral sequence are $\mathbb{Q}[t]$-linear, we can evaluate the terms $E_{r}, r \geq 2$, at $t=1$ and because evaluation at $t=1$ commutes with taking homology with respect to each $\delta_{r}$ (see [1], Lemma (A.7.2)), we get a converging spectral sequence $\left(\bar{E}_{r}, \bar{\delta}_{r}\right)$, where

$$
\bar{E}_{r}=\left(E_{r}\right)_{t=1}
$$

and $\bar{\delta}_{r}$ is induced by $\delta_{r}$. This spectral sequence is concentrated in the first column and $\bar{E}_{\infty}^{*, *}$ is (noncanonically and not preserving the grading) isomorphic to $H^{*}\left(X_{G} ; \mathbb{Q}\right)_{t=1}$ as a $\mathbb{Q}$-vector space. By the evaluation theorem (cf. [1], Theorem (3.5.1)), we have a canonical isomorphism (of ungraded modules)

$$
H\left(X_{G} ; \mathbb{Q}\right)_{t=1} \cong H\left(X^{G} ; \mathbb{Q}\right) \text {. }
$$

Because $X$ fulfills Poincaré duality over $\mathbb{Q}$, the term $\bar{E}_{2}^{*, *}=\bar{E}_{2}^{0, *}$ is an $\mathbb{N}$-graded $\mathbb{Q}$-Poincaré duality algebra of formal dimension $2 m+1$, where we use the grading induced by $E_{2}$. As $X^{G} \neq \emptyset$, we have $\bar{E}_{r} \neq 0$ for all $r$. Now the claim follows from Propositions 1 and 2 in the second section of this paper.

\section{Proof of Theorem 2}

Let $p$ be an odd prime number, let $G=\mathbb{Z} / p$ and let $X$ be a finite dimensional connected $G$-CW complex that is an $\mathbb{F}_{p}$-PD space of formal dimension $n$. Furthermore, we assume that $\beta\left(H^{*}\left(X ; \mathbb{F}_{p}\right)\right)=0$, where

$$
\beta: H^{*}\left(X ; \mathbb{F}_{p}\right) \rightarrow H^{*+1}\left(X ; \mathbb{F}_{p}\right)
$$

is the Bockstein operator associated to the exact sequence of coefficients

$$
0 \rightarrow \mathbb{Z} / p \rightarrow \mathbb{Z} / p^{2} \rightarrow \mathbb{Z} / p \rightarrow 0
$$

This condition is equivalent to the requirement that each cohomology class in $H^{*}\left(X ; \mathbb{F}_{p}\right)$ can be lifted to a cohomology class in $H^{*}\left(X ; \mathbb{Z} / p^{2}\right)$, which is the case, if and only if $H^{*}\left(X ; \mathbb{Z}_{(p)}\right)$ does not contain a direct summand of the form $\mathbb{Z} / p$. Note that the $G$-action on $X$ induces a $\mathbb{Z} / p^{2}[G]$-module structure on $H^{*}\left(X ; \mathbb{Z} / p^{2}\right)$. By our assumption on $\beta, H^{*}\left(X ; \mathbb{Z} / p^{2}\right)$ is a free $\mathbb{Z} / p^{2}$-module, so the following proposition is an immediate consequence of [6], Proposition 6 , which is shown using a little representation theory over $\mathbb{Z} / p^{2}$. 
Proposition 4. For each $i$ there is an $\mathbb{F}_{p}[G]$-linear decomposition

$$
H^{i}\left(X ; \mathbb{F}_{p}\right)=H^{i}\left(X ; \mathbb{Z} / p^{2}\right) \otimes \mathbb{F}_{p} \cong T^{i} \oplus F^{i} \oplus R^{i},
$$

where $T^{i}$ is a trivial $\mathbb{F}_{p}[G]$-module, $F^{i}$ is a direct sum of free $\mathbb{F}_{p}[G]$-modules and $R^{i}$ is a direct sum of modules of the form $\operatorname{ker} \epsilon$, where $\epsilon: \mathbb{F}_{p}[G] \rightarrow \mathbb{F}_{p}$ is the augmentation map.

Let $\left(E_{r}, \delta_{r}\right)$ denote the cohomological Leray-Serre spectral sequence for the Borel fibration

$$
X \rightarrow X_{G}=E G \times{ }_{G} X \rightarrow B G
$$

with coefficients in $\mathbb{F}_{p}$. For $r \geq 2$, each $E_{r}$ is a differential bigraded algebra over $H^{*}\left(B G ; \mathbb{F}_{p}\right) \cong \mathbb{F}_{p}[t] \otimes \Lambda(s)$, where $t \in H^{2}\left(B G ; \mathbb{F}_{p}\right)$ and $s \in H^{1}\left(B G ; \mathbb{F}_{p}\right)$ are generators and $\beta(s)=t$. By the evaluation theorem (cf. 11, Theorem (1.4.5)), the inclusion $X^{G} \hookrightarrow X$ induces an isomorphism of (ungraded) $\Lambda(s)$-algebras

$$
H\left(X_{G} ; \mathbb{F}_{p}\right)_{t=1} \cong H\left(X^{G} ; \mathbb{F}_{p}\right) \otimes \Lambda(s),
$$

hence, after evaluating at $s=0$, we get an induced isomorphism

$$
H\left(X_{G} ; \mathbb{F}_{p}\right)_{t=1, s=0} \cong H\left(X^{G} ; \mathbb{F}_{p}\right) .
$$

Now we encounter the following difficulty that did not arise in the consideration of $S^{1}$-actions above: In order to calculate the dimension of the left-hand side of the second isomorphism one has to get around the difficulty that evaluation at $s=0$ does not commute with taking homology in general, and so a spectral sequence argument as in Section 3 does not seem to be feasible in this case. However, under the additional assumption $\beta=0$, we can get around this difficulty by using the following fact (cf. [6], Proposition 9, and the last part of the proof of Proposition $10)$.

Proposition 5. For all $r \geq 2$, the localized terms $E_{r}\left[t^{-1}\right]$ are finitely generated free $(\mathbb{Z} \times \mathbb{N})$-bigraded differential $\Lambda(s) \otimes \mathbb{F}_{p}\left[t, t^{-1}\right]$-algebras. Furthermore, evaluation at $t=1$ and $s=0$ on $E_{r}\left[t^{-1}\right]$ commutes with taking homology with respect to the differentials induced by $\delta_{r}$.

Now, we set

$$
\bar{E}_{r}=\left(E_{r}\right)_{t=1, s=0}
$$

and use the induced differentials $\bar{\delta}_{r}$. Each term $\bar{E}_{r}$ has an induced $(\mathbb{Z} / 2 \times \mathbb{N})$-grading and for $r=2$ we obtain

$$
\bar{E}_{2}^{\gamma, \mu} \cong H^{\gamma}\left(G ; H^{\mu}\left(X ; \mathbb{F}_{p}\right)\right)_{t=1, s=0} \cong\left\{\begin{array}{l}
\mathbb{F}_{p}^{\operatorname{dim} T^{\mu}}, \text { if } \gamma \text { is even, } \\
\mathbb{F}_{p}^{\frac{\operatorname{dim} R^{\mu}}{p-1}}, \text { if } \gamma \text { is odd }
\end{array}\right.
$$

Here, we used Proposition 4 and the fact (which follows from usual dimension shifting) that $H^{i}\left(\mathbb{F}_{p} ; \operatorname{ker} \epsilon\right)\left[t^{-1}\right]$ is isomorphic to $\mathbb{F}_{p}$ in odd degrees and is equal to zero in even degrees. Further, we get from Proposition 5 and the evaluation theorem that

$$
\operatorname{dim}_{\mathbb{F}_{p}} \bar{E}_{\infty}=\frac{1}{2} \operatorname{dim}_{\mathbb{F}_{p}}\left(E_{\infty}\right)_{t=1}=\operatorname{dim}_{\mathbb{F}_{p}} H^{*}\left(X^{G} ; \mathbb{F}_{p}\right) .
$$

Now, the proof of Theorem 3 is completed by using induction in the spectral sequence. Notice that $\bar{E}_{2}^{*, *}$ is a $(\mathbb{Z} / 2 \times \mathbb{N})$-bigraded connected Poincaré duality algebra over $\mathbb{F}_{p}$ of formal dimension $n$ in the sense of Definition 1 (cf. [6], proof of Proposition 9). For the induction step, if $n$ is even, we use Lemma 1 and the fact that 
the Euler characteristic of a $\mathbb{Z} / 2$-graded differential complex does not change after taking homology with respect to a differential of odd degree. If $n$ is odd, we use Proposition 1 and Proposition 2

\section{EXAMPLES, APPLICATIONS AND CONCLUDING REMARKS}

The following examples illustrate the significance of the conditions on the Betti numbers in the second part of Theorems 1 and 2. The example of free $S^{1}$ or $\mathbb{Z} / p$ actions on spheres of odd dimension shows that the requirement $X^{G} \neq \emptyset$ in the second part of Theorem 1 and in the second part of Theorem 2 is necessary. In [4], p. 425, an example of an $S^{1}$-action on $X=S^{3} \times S^{5} \times S^{9}$ is constructed whose fixed point set is an $S^{7}$-bundle over $S^{3} \times S^{5}$ and has total Betti number 6 (with coefficients in $\mathbb{Q}$ ). As the total Betti number of $X$ is 8 , one sees that even if fixed points exist, the assumption on the vanishing of certain Betti numbers in the second part of Theorem 1 is needed. Restricting this $S^{1}$-action to $\mathbb{Z} / p \subset S^{1}$, we similarly may conclude that all additional assumptions in the second part of Theorem 2 are necessary.

Now we will construct examples of Poincaré duality spaces of odd formal dimension that fulfill the additional requirement on Betti numbers in the second part of Theorems 1 and 2, Let $X$ be an arbitrary connected finite simplicial complex with the property that its even dimensional integral cohomology is concentrated in degree 0 . Now embed $X$ in a Euclidean space $\mathbb{R}^{2 m+1}$, where $2 m+1 \geq 2 \operatorname{dim} X+1$, and take a regular neighbourhood $R$ of $X$ inside $\mathbb{R}^{2 m+1}$ which can be assumed to be a compact oriented smooth manifold with boundary. Gluing two copies of this manifold (the orientation of one of which had been reversed) along their boundaries yields a $(2 m+1)$-dimensional connected oriented smooth manifold $Y$, whose even dimensional integral cohomology below dimension $m+1$ is concentrated in degree 0 . This follows from the Mayer-Vietoris sequence and the fact that by general position, the inclusion $\partial R \hookrightarrow R$ induces isomorphisms of homotopy groups up to degree $2 m+1-\operatorname{dim} X-2 \geq m-1$ and a surjection in degree $2 m+1-\operatorname{dim} X-1 \geq m$. In particular, the connecting homomorphism $H^{i}(\partial R ; \mathbb{Z}) \rightarrow H^{i+1}(Y ; \mathbb{Z})$ in the MayerVietoris sequence is 0 , if $i \leq m-1$. Hence, for actions on $Y$, the second part of Theorems 1 and 2 can be applied (assuming that the induced action on $H^{*}\left(Y ; \mathbb{F}_{p}\right)$ is trivial in the case of $G=\mathbb{Z} / p$ ).

Another example illustrating the second part of Theorem 1 and Theorem 2 can be constructed as follows. Consider the space $X=S^{1} \times S^{2 m}$ equipped with the $S^{1}$ action that acts trivially on the first factor and is the usual rotation action (fixing north and south pole) on the second factor. The fixed point set of this $S^{1}$-action is the union of two circles. Next we choose one point in each fixed point component and remove small $S^{1}$-invariant neighborhoods equivariantly diffeomorphic to $D^{2 m+1}$ (with a linear $S^{1}$-action) around each of these two fixed points which gives an $S^{1}$-manifold $Z$ with two boundary components each of which is equivariantly diffeomorphic to $S^{2 m}$ with the rotation action by $S^{1}$. Now we form the equivariant connected sum of $Z$ and $[0,1] \times S^{2 m}$, where on the last space $S^{1}$ acts trivially on the first factor and by a rotation action on the second. In this way, we obtain a $(2 m+1)$-dimensional oriented $S^{1}$-manifold which fulfills the requirement of the second part of Theorems 10 and $\left[2\right.$ (using the induced action by $\mathbb{Z} / p \subset S^{1}$ ). It is easy to check that the integral cohomology of this space has rank 1 in degrees 0 and $2 m+1$, rank 2 in degrees 1 and $2 m$ and is 0 in all other degrees. The total 
Betti number of the fixed set (which is just a single copy of $S^{1}$ ) is two. So the Leray-Serre spectral sequence for the Borel construction does not collapse at the $E_{2}$-level in this case.

In this paper we have been working in the category of $G$-CW complexes. But using Čech cohomology and the usual somewhat more technical machinery (see, e.g. 1]), one can extend all the results to general $G$-spaces which fulfill the hypothesis (LT) for the localization theorem (see [1, p. 208). As this generalization is straightforward, we leave it to the interested reader.

\section{REFERENCES}

[1] Ch. Allday, V. Puppe, Cohomological methods in transformation groups, Cambridge University Press, 1993 MR 94g:55009

[2] T. Chang, T. Skjelbred, Group actions on Poincaré duality spaces, Bull. Amer. Math. Soc. 78 (1972), 1024-1026 MR 46:6346

[3] G.E. Bredon, Fixed point sets of actions on Poincaré duality spaces, Topology 12 (1973), 159-175 MR 48:9708

[4] G.E. Bredon, Introduction to compact transformation groups, Academic Press, 1972 MR 54:1265

[5] B. Hanke, Poincaré duality and deformations of algebras, Contemp. Math. 279 (2001), 129-133 MR 2002k:57056

[6] B. Hanke, Inner products and $\mathbb{Z} / p$-actions on Poincaré duality spaces, Forum Mathematicum (to appear) http://www.mathematik.uni-muenchen.de/ hanke

[7] B. Hanke, Actions of finite p-groups on homology manifolds, Math. Proc. Camb. Phil. Soc. 131 (2001), 473-486 MR 2002j:57065

[8] M. Raussen, Rational cohomology and homotopy of spaces with circle action, LNM 1509 (1992), 313-325, Springer-Verlag MR 93h:57052

[9] A. Sikora, Torus and $\mathbb{Z} /$ p-actions on manifolds, Topology (to appear) http: //www.crm.umontreal.ca/ sikora

Department of Mathematics, University of Hawait, 2565 Mc Carthy Mall, Honolulu, HAWAII 96822

E-mail address: chris@math.hawaii.edu

Department of Mathematics, Universität München, Theresienstr. 39, 80333 München, Germany

E-mail address: hanke@rz.mathematik.uni-muenchen.de

Department of Mathematics, Universität Konstanz, 78457 Konstanz, Germany

E-mail address: Volker.Puppe@uni-konstanz.de 